\title{
Validation of an Improved Patient-Specific Mold Design for Registration of In-Vivo MRI and Histology of the Prostate
}

\author{
An Elen ${ }^{1}$, Sofie Isebaert ${ }^{1}$, Frederik De Keyzer ${ }^{2}$, Uwe Himmelreich ${ }^{3}$, Steven \\ Joniau $^{4}$, Lorenzo Tosco ${ }^{4}$, Wouter Everaerts ${ }^{4}$, Tom Dresselaers ${ }^{3}$, Evelyne \\ Lerut $^{3}$, Raymond Oyen ${ }^{3,2}$, Roger Bourne ${ }^{5}$, Frederik Maes ${ }^{6}$, and Karin \\ Haustermans ${ }^{1}$ \\ 1 Lab. of Exp. Radiotherapy, Dept. of Oncology, KU Leuven, Leuven, Belgium \\ 2 Dept. of Radiology, University Hospitals Leuven, Leuven, Belgium \\ 3 Dept. of Imaging and Pathology, University Hospitals Leuven, Leuven, Belgium \\ 4 Dept. of Urology, University Hospitals, Leuven, Belgium \\ 5 Discipline of Medical Radiation Sciences, University of Sydney, Australia \\ ${ }^{6}$ Dept. of Electrical Engineering (ESAT/PSI), KU Leuven, Leuven, Belgium
}

\begin{abstract}
Fusion of in-vivo magnetic resonance imaging (MRI) with whole mount histology of the prostate is facilitated by the use of a patient-specific mold, that is designed from in-vivo MRI. The mold defines specific sectioning planes with the same orientation and position relative to the prostate as the stack of MRI slices, reducing the registration problem from a $3 \mathrm{D}$ to a $2 \mathrm{D}$ problem. We present an innovative mold design that specifies the in- and outflow of the urethra as additional landmarks for correct positioning of the prostate in the mold, that allows for the fresh prostate tissue to be fixated inside the mold such that its in-vivo shape is maintained, and that allows for ex-vivo MRI of the prostate in the mold in alignment with in-vivo MRI using the mold as reference frame. Using high-resolution 3D ex-vivo MRI aligned with in-vivo MRI, we demonstrate that our improved mold design results in a more accurate positioning of the prostate inside the mold, significantly reducing out-of-plane rotational offsets. Initial results show that the proposed workflow has the potential to provide detailed histopathological ground truth for the quantitative interpretation of in-vivo and ex-vivo MRI in prostate cancer.
\end{abstract}

\section{Introduction}

Radiation therapy treatment of prostate cancer is evolving towards focal boosting strategies, to maximize both tumor control and normal tissue sparing. To this end, accurate identification of the exact location and boundaries of the tumor are of utmost importance, but so far, there is no consensus on the ideal MRI sequence to do so. Validation against gold standard whole mount pathology is therefore necessary, but this is not trivial. Several approaches for comparing imaging results with histological findings have been used in literature, going 
from lesion- or sector-based analyses [1-3] over visual comparison or direct registration of the 3D MRI volume with the 2D histological slices [4]. Histology to in-vivo MRI registration is a complicated problem due to the typically low outof-plane resolution of both sets of images, possible deformation of the histology slices and the limited amount of detail in the in-vivo MRI images, which makes it hard to find corresponding tissue points in both images. Reconstruction of the histology slices into a 3D stack makes the registration problem of histology to in-vivo MRI a 3D problem [5, 6]. However, due to the low out-of-plane resolution and resulting sparse 3D data, the registration problem is usually reduced to a set of $2 \mathrm{D}$ problems, by visually $[7,8]$ or automatically [9] selecting the most corresponding MRI slice for every histology image. Of course, this is only valid if the orientation of the MRI planes is identical to that of the histological sections. To ensure this, several prostate sectioning devices have been developed. Some require visual orientation and unconstrained positioning of the prostate in the device $[7,10]$. Others use a mold with a patient-specific prostate shaped cavity to position the sectioning planes relative to the prostate $[11,12]$. This would result in precisely corresponding $2 \mathrm{D}$ histology and MRI slices, provided that the prostate specimen is correctly positioned inside the mold. Using an innovative mold design that enables high-resolution, high-field strength 3D ex-vivo MRI of the prostate tissue inside the mold, we show that this is not guaranteed and that significant out-of-plane rotational offsets may occur despite the use of the mold. The presented improved mold design uses additional landmarks to prevent such mispositioning. Ex-vivo MRI is not only valuable for validation purposes, but also simplifies the histology to in-vivo MRI registration workflow.

\section{Registration Workflow and Improved Mold Design}

Figure 1 shows an overview of the workflow to register histology to in-vivo MRI, in order to obtain a gold standard for tumor localization and delineation in in-vivo MRI, using high-resolution ex-vivo MRI as intermediate reference to facilitate the registration.

An in-vivo T2-weighted MRI image stack is acquired with similar orientation and slice spacing as preferred for histological sectioning, in our case typically para-axial orthogonal to the urethra outflow. (Para-) sagittal and coronal T2-weighted image stacks are acquired as well and the prostate is manually delineated in all 3 stacks simultaneously, by visualizing the intersection of each contour drawn in any image slice with all slices in the other stacks, such that a consistent 3D delineation is obtained from which the prostate shape is reconstructed. Next, a mold template, consisting of left and right halves, with predefined parallel cutting slots is positioned around the prostate shape such that the cutting slots exactly coincide with the imaging planes. A patient-specific mold is obtained by subtracting the prostate shape from the mold template, and is then $3 \mathrm{D}$ printed.

We present an improved mold design with the following main and unique features as illustrated in Figure 2: 

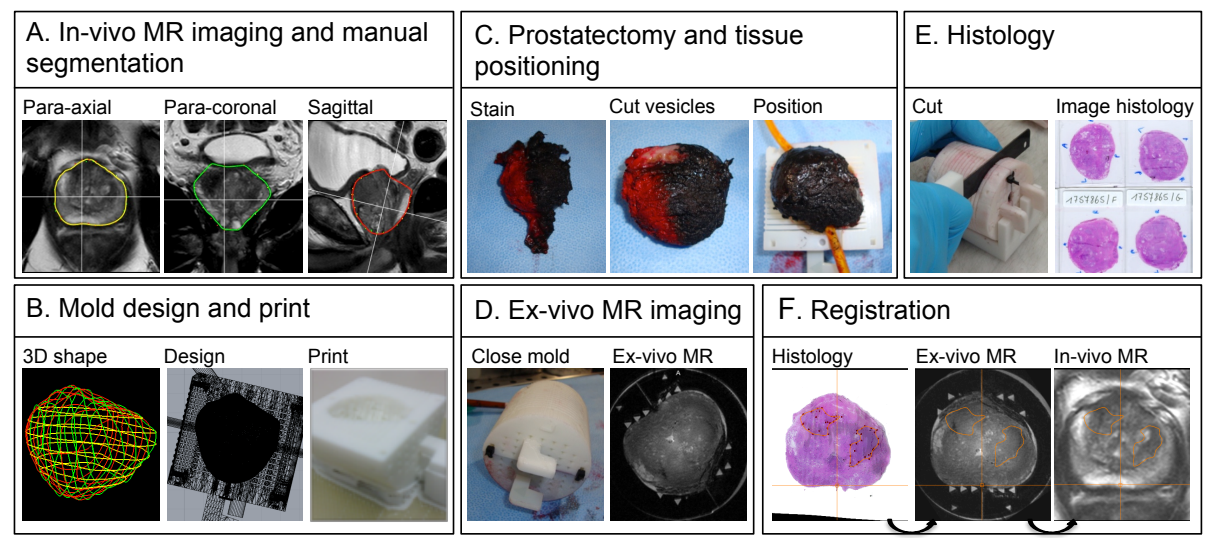

Fig. 1. Overview of the entire workflow. In-vivo MRI images are manually segmented to design a patient-specific mold, in which the prostate tissue is positioned after prostatectomy. Ex-vivo MRI is acquired of the prostate while in the mold, before cutting the tissue and acquiring histology images. The ex-vivo MRI stack is used as high-detail intermediate image to facilitate the histology to in-vivo MRI registration.

1. The position and direction of the in- and outflow of the urethra at the prostate surface are indicated in the in-vivo MRI images and are replicated in the mold as tube shaped openings. These are used as guides for a urethra catheter when positioning the prostate in the mold.

2. Infiltration holes are added to the mold, allowing the tissue to be fixated while in the mold. By placing the prostate in the mold prior to fixation, it is fixated in its in-vivo shape, minimizing possible deformation of the tissue [13] and minimizing positioning difficulties due to tissue fixation-induced rigidity [11].

3. The overall shape and size of the mold are adjusted to fit exactly in the bore of a high-field strength $(9.4 \mathrm{~T})$ preclinical MRI scanner with $72 \mathrm{~mm}$ inside diameter, while contained in a Plexiglas container tube filled with formalin. Hence, high-resolution ex-vivo MRI can be obtained of the prostate in the mold. Several landmarks that are fluid filled when the mold is immersed, are visible during ex-vivo scanning and enable the positioning of the ex-vivo MRI planes in correspondence with the in-vivo MRI planes.

After prostatectomy, the prostate is stained such that the right and left half can be distinguished, the seminal vesicles are removed and a catheter is inserted through the urethra. The prostate is then positioned in the mold guided by the catheter holes. After closing the mold with straps, the catheter is removed and the tissue is fixated overnight using formalin, while in the mold. The mold is then placed in a tubular container filled with formalin and imaged with highfield ex-vivo MRI, to obtain high-detail images. A stack of T2-weighted images is acquired at the location of the cutting slots of the mold, thus corresponding to the in-vivo MRI slices. 


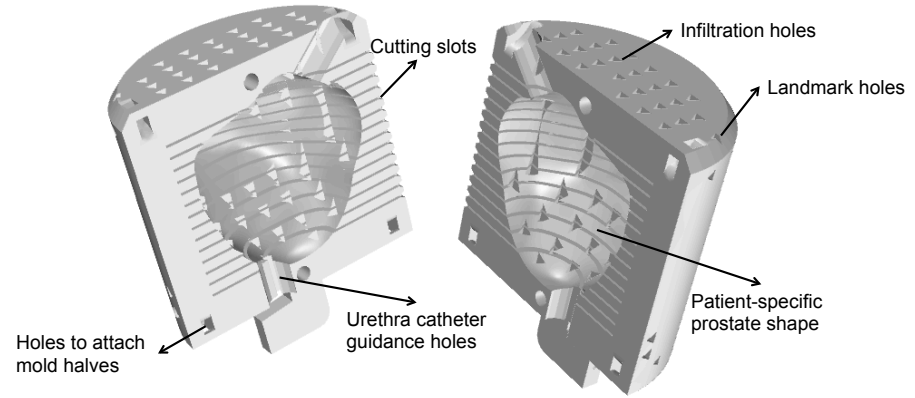

Fig. 2. Proposed patient-specific prostate mold design with indication of the main features.

After ex-vivo imaging, the prostate specimen is sliced inside the mold using the cutting slots and processed using the standard pathological workup. The thick sections are embedded in paraffin and thin sections are sliced from these macro blocks using a microtome. The thin sections are then hematoxylin-eosin (H\&E) stained and imaged using a flatbed scanner.

The registration of the stained histology slices to in-vivo MRI is then addressed in two steps, using ex-vivo MRI as intermediate reference. First, the in- and ex-vivo MRI images are rigidly registered, assuming possible ex-vivo shrinking of the tissue to be negligible at this stage [11] and geometric distortion of the ex-vivo MRI images to be limited. As the mold was created from in-vivo MRI delineations, it can be directly overlaid on the in-vivo MRI image, reducing the ex- to in-vivo MRI registration to the simple matching of the mold shape in both image stacks. Secondly, a slice-by-slice 2D non-rigid registration is performed to align the histology and ex-vivo MRI image slices, guided by the many tissue features that are visible in both high-detail images. The Elastix registration package [14] is used for all registrations.

\section{Validation of mold design}

\subsection{Image Acquisition}

This study was approved by the local Institutional Review Board and written informed consent was obtained from all patients. Six patients with biopsy proven prostate cancer who were scheduled for robotic assisted laparoscopic prostatectomy were included in this study. They all had Gleason score 6 or 7 and different clinical T-stages.

In-vivo imaging was performed on a $1.5 \mathrm{~T}$ (Siemens SonataVision, Patients 1-3) or 3T (Philips Ingenia, Patients 4-6) MRI unit. The prostate was delineated by an experienced radiologist in 3 orthogonal T2-weighted in-vivo MRI stacks with a resolution of $0.47 \times 0.47 \times 3 \mathrm{~mm}$ and a patient-specific mold was designed for each patient and printed using a desktop PP3D UP! plus 3D printer. For Patients 1-3 the presented mold design, but without catheter guidance holes was 
used to simulate classic mold designs, while for Patients 4-6 the presented mold design with catheter holes was used.

Ex-vivo imaging of the prostate specimen inside the mold was performed using a 9.4T Biospec MRI scanner (Bruker Biospin, Ettlingen, Germany). Apart from a stack of $2 \mathrm{D}$ slices aligned with the cutting slots of the mold with a resolution of $0.25 \times 0.25 \times 3 \mathrm{~mm}$, a T2-weighted 3D image with high isotropic resolution of $0.18 \mathrm{~mm}$ was acquired as well, to validate the mold design.

The H\&E stained histology slices were imaged using a flatbed scanner with a resolution of $0.042 \times 0.042 \mathrm{~mm}$.

\subsection{Evaluation}

The correspondence between in-vivo MRI and histology is based on the assumption that the prostate specimen is correctly positioned in the mold. To evaluate the validity of this assumption, we manually indicated corresponding tissue landmarks in the 3D ex-vivo MRI image and in-vivo MRI image stacks. Both image sets were rigidly aligned solely based on the mold itself and the same transformation was applied to the landmarks. The residual misalignment between them after mold-based registration was visually assessed and quantified by the rigid transformation $T_{r}$. The rotation angles derived from $T_{r}$, i.e. the angular offsets between the in-vivo situation and the position of the prostate specimen inside the mold, which ideally should be zero, are summarized in Table 1.

For all 3 cases for which a mold without catheter guides was used (Patients 1-3), significant out-of-plane rotations up to almost 35 degrees were found, especially around the left/right axis. For a slice spacing of $3 \mathrm{~mm}$ and a typical prostate extent of $4 \mathrm{~cm}$, a mispositioning of the specimen by 8 degrees around the center already results in tissue displacements of 1 slice. Figure 3 shows the mold-based alignment of the 3D ex-vivo MRI with the 3 orthogonal in-vivo MRI stacks of Patient 1, as well as their corrected landmark-based alignment, illustrating the rotational mispositioning of the specimen in the mold.

For all 3 cases for which a mold with catheter guides was used (Patients 4-6), out-of-plane rotations were smaller than 3 degrees, resulting in maximal tissue

\begin{tabular}{|l||c|c||c|c|c|}
\hline & $\begin{array}{c}\text { Prostate } \\
\text { volume [cc] }\end{array}$ & $\begin{array}{c}\text { Catheter } \\
\text { guides }\end{array}$ & $\begin{array}{c}\text { Left-right } \\
\text { (out-of-plane) }\end{array}$ & $\begin{array}{c}\text { Antero-posterior } \\
\text { (out-of-plane) }\end{array}$ & $\begin{array}{c}\text { Cranio-caudal } \\
\text { (in-plane) }\end{array}$ \\
\hline \hline Patient 1 & 50.1 & No & 12.3 & -6.2 & 3.9 \\
\hline Patient 2 & 31.2 & No & 34.6 & 0.7 & 7.4 \\
\hline Patient 3 & 24.6 & No & 12.2 & -0.4 & -2.4 \\
\hline Patient 4 & 56.6 & Yes & 2.0 & -2.2 & -10.4 \\
\hline Patient 5 & 47.8 & Yes & 1.6 & -1.2 & 1.9 \\
\hline Patient 6 & 49.3 & Yes & -1.6 & 2.7 & 1.2 \\
\hline
\end{tabular}

Table 1. Residual rotational offsets [in degrees] around each axis for mold-based registration between in-vivo and ex-vivo MRI as determined using internal tissue landmarks, for 3 cases without and 3 cases with catheter guidance embedded in the mold. 
displacements of less than half a slice. For Patient 4, an in-plane rotational offset of around 10 degrees was found. For Patients 5 and 6 , care was taken to position the cutting plane of the seminal vesicles symmetrical in both mold halves to prevent in-plane rotation.

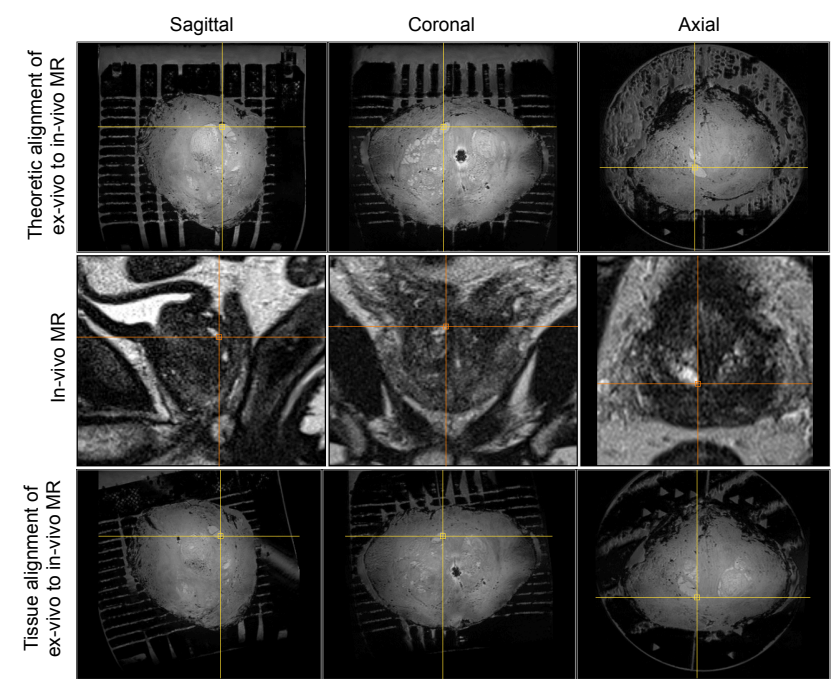

Fig. 3. Alignment of the 3 orthogonal in-vivo MRI stacks (middle row) of Patient 1 to the $3 \mathrm{D}$ ex-vivo image of the prostate specimen solely based on the mold (top row) and on internal tissue landmarks (bottom row), illustrating significant residual rotational misalignment for mold-based registration.

This quantifies the ex- to in-vivo MRI registration. To validate the second part of the registration workflow, non-rigidly registering histology to ex-vivo MRI, we manually indicated corresponding landmarks in every high-detail histology and ex-vivo MRI slice. The histology landmarks were transformed to the ex-vivo MRI using the found transformation field and the mean distance between both sets of landmarks was calculated to be $0.90 \pm 0.56$ and $0.94 \pm 0.44 \mathrm{~mm}$ for Patients 5 and 6 respectively (the correctly positioned specimens).

\section{Discussion and Conclusion}

We presented an innovative mold design to support the pathological workup of the prostate without altering it and to facilitate the subsequent registration of the histological slices to in-vivo MRI. The unique features of our design include infiltration holes allowing fixation of the fresh tissue in the mold to retain its in-vivo shape, a cylindrical outer shape that fits inside the bore of a high-field strength MRI scanner, liquid filled channels to provide MR-visible landmarks and catheter holes to provide extra guidance for more accurate specimen positioning. 
This improved mold design offers the possibility to scan the prostate inside the mold, after or prior to fixation, using high resolution ex-vivo MRI, such that corresponding in-vivo MRI, ex-vivo MRI and histology slices are obtained with the same orientation and position relative to the prostate specimen. This opens many opportunities for addressing a variety of research questions.

First, ex-vivo MRI can be used as intermediate reference for histology to invivo MRI registration. The high-detail ex-vivo MRI images are easily registered to the in-vivo MRI images based on the mold and provide a rich set of features to guide the non-rigid registration of the histology slices with ex-vivo MRI slices. As a proof-of-concept, the accuracy of histology to ex-vivo MRI and ex- to in-vivo MRI registration was quantified for 2 patients.

Secondly, the possibility to scan the prostate inside the mold with high 3D resolution and contrast allows to validate the underlying assumption of moldbased registration that the patient-specific shape of the mold uniquely constrains the positioning of the specimen inside the mold. To the best of our knowledge, this has not yet been validated despite the frequent use of such molds.

Based on the alignment of internal tissue landmarks as visible in 3D ex-vivo MRI on the one hand and multi-planar in-vivo MRI stacks on the other hand, we showed that the traditional mold design may still result in significant rotational inaccuracies in the positioning of the tissue. This may be attributed to various factors that all introduce some residual degrees of freedom when positioning the specimen inside the mold. First of all, the prostate shape is rather simple and somewhat cylindrically symmetric with little local features. Secondly, the shape of the mold may deviate from that of the excised prostate specimen due to uncertainties in the delineation and during the prostatectomy itself, such that both may not be perfectly fitting. Thirdly, as the tissue is placed inside the mold prior to fixation, it is slightly deformable.

We showed that these residual degrees of freedom could be substantially reduced by extending the mold with catheter guides at the location and with the orientation of the urethra in- and outflow of the prostate. By inserting a catheter through the urethra, the positioning of the specimen inside the mold is further restricted by its in- and outflow location on the prostate surface. By additionally taking care that the plane, along which the seminal vesicles were cut, is placed symmetrically in both mold halves, precise positioning of the tissue in the mold is achieved with residual rotational inaccuracies smaller than 3 degrees.

More accurate positioning of the specimen inside the mold and the availability of high resolution ex-vivo MRI both contribute to improving the registration accuracy between histology and in-vivo MRI. In the end, this will help to evaluate the potential of different in-vivo and ex-vivo MRI techniques for tumor detection and delineation and to correlate quantitative image features with the underlying (patho-)physiology based on ground truth as provided by histopathology. This will improve the predictive value of MRI and our understanding of its current limitations. Vice versa, the fusion with in-vivo and ex-vivo MRI could be used by pathologists to guide the quick assessment of suspect tissue regions. 


\section{References}

1. Haider, M.A., van der Kwast, T.H., Tanguay, J., Evans, A.J., Hashmi, A.T., Lockwood, G., Trachtenberg, J.: Combined T2-weighted and diffusion-weighted MRI for localization of prostate cancer. AJR 189 (2007) 323-328

2. Turkbey, B., Pinto, P.A., Mani, H., Bernardo, M., Pang, Y., McKinney, Y.L., Khurana, K., Ravizzini, G.C., Albert, P.S., Merino, M.J., Choyke, P.L.: Prostate cancer: value of multiparametric MR imaging at $3 \mathrm{~T}$ for detection - histopathologic correlation. Radiology 255 (2010) 89-99

3. Isebaert, S., Van den Bergh, L., Haustermans, K., Joniau, S., Lerut, E., De Wever, L., De Keyzer, F., Budiharto, T., Slagmolen, P., Van Poppel, H., Oyen, R.: Multiparametric MRI for prostate cancer localization in correlation to whole-mount histopathology. JMRI 37 (2013) 1392-1401

4. Meyer, C., Ma, B., Kunju, L.P., Davenport, M., Piert, M.: Challenges in accurate registration of 3-D medical imaging and histopathology in primary prostate cancer. Eur J Nucl Med Mol Imaging 40 (Suppl 1) (2013) S72-S78

5. Bart, S., Mozer, P., Hemar, P., Lenaour, G., Comperat, E., Renard-Penna, R., Chartier-Kastler, E., Troccaz, J.: MRI-histology registration in prostate cancer. In: Surgetica2005, Merloz P., Troccaz J. (Eds), Sauramps Medical. (2005) 361-367

6. Orczyk, C., Mikheev, A., Rosenbrantz, A.B., Melamed, J., Taneja, S.S., Rusinek, H.: Imaging of prostate cancer: a platform for $3 \mathrm{D}$ co-registration of in-vivo MRI ex-vivo MRI and pathology. Proc. of SPIE Int soc Opt Eng 8316 (2012)

7. Kalavagunta, C., Zhou, X., Schmechel, S.C., Metzger, G.J.: Registration of in vivo prostate MRI and pseudo-whole mount histology using local affine transformations guided by internal structures (LATIS). JMRI 41 (2015) 1104-1114

8. Chappelow, J., Bloch, B.N., Rofsky, N., Genega, E., Lenkinski, R., DeWolf, W., Madabhushi, A.: Elastic registration of multimodal prostate MRI and histology via multiattribute combined mutual information. Med. Phys. 38(4) (2011) 2005-2018

9. Xiao, G., Bloch, B.N., Chappelow, J., Genega, E.M., Rofsky, N.M., Lenkinski, R.E., Tomaszewski, J., Feldman, M.D., Rosen, M., Madabhushi, A.: Determining histology-MRI slice correspondences for defining MRI-based disease signatures of prostate cancer. Comput Med Imag Grap 35 (2011) 568-578

10. Hong Chen, L., Ho, H., Lazaro, R., Hua Thng, C., Yuen, J., Sing Ng, W., Cheng, C.: Optimum slicing of radical prostatectomy specimens for correlation between histopathology and medical images. Int J CARS 5 (2010) 471-487

11. Shah, V., Pohida, T., Turkbey, B., Mani, H., Merino, M., Pinto, P.A., Choyke, P., Bernardo, M.: A method for correlating in vivo prostate magnetic resonance imaging and histopathology using individualized magnetic resonance-based molds. Rev Sci Instrum 80(10) (2009) 104301

12. Priester, A., Natarajan, S., Le, J.D., Garritano, J., Radosavcev, B., Grundfest, W., Margolis, D.J., Marks, L.S., Huang, J.: A system for evaluating magnetic resonance imaging of prostate cancer using patient-specific $3 \mathrm{D}$ printed molds. Am J Clin Exp Urol 2(2) (2014) 127-135

13. Orczyk, C., Taneja, S.S., Rusinek, H., Rosenkrantz, A.B.: Assessment of change in prostate volume and shape following surgical resection through co-registration of in-vivo MRI and fresh specimen ex-vivo MRI. Clin Radiol 69(10) (2014) 398-403

14. Klein, S., Staring, M., Murphy, K., Viergever, M., Pluim, J.: Elastix: a toolbox for intensity based medical image registration. IEEE TMI 29(1) (2010) 196-205 